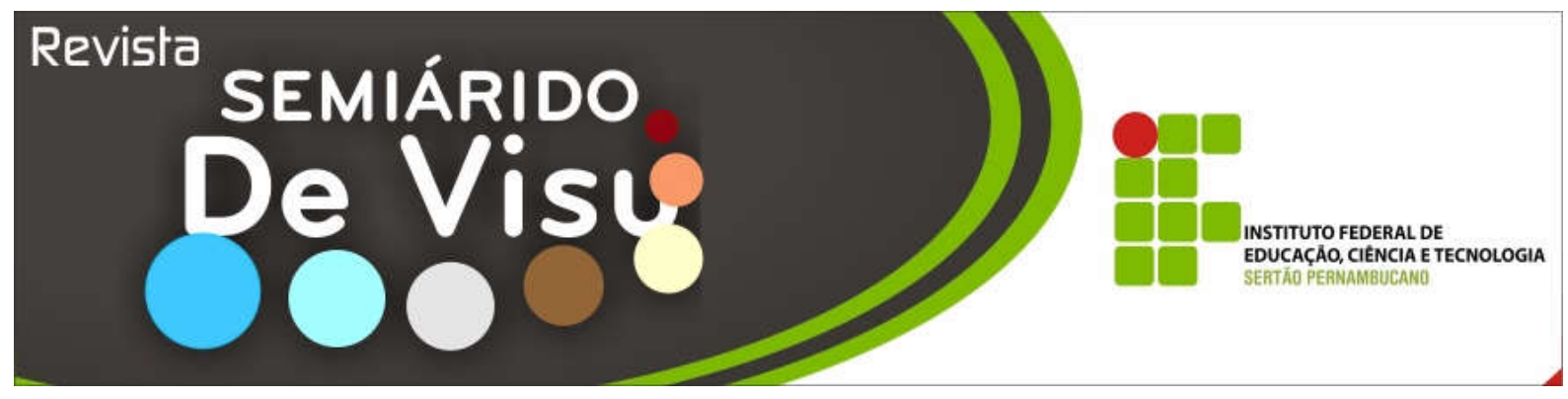

\title{
Aceitabilidade e adesão à alimentação escolar por estudantes do IF Sertão Pernambucano - campus Salgueiro
}

\author{
Nataly Roberta Bezerra Santana Carlini ${ }^{1}$, Fernanda Fernandes Pinheiro da Costa $^{2}$, Roberta Verônica dos Santos \\ Carvalho Mesquita ${ }^{3}$ \\ 1,2,3Ins tituto Federal de Educação, Ciência e Tecnologia do Sertão Pernambucano - IF SERTÃO-PE, Campus \\ Salgueiro, Rodovia BR 232, Km 508, sentido Recife, Zona Rural - Salgueiro-PE-Brasil, CEP: 56.000-000, E-mail: \\ 1nataly.santana@ifsertao-pe.edu.br; ${ }^{2}$ fernanda.fernandes@ifsertao-pe.edu.br; ${ }^{3}$ rob erta.mesquita@ifsertao-pe.edu.br
}

RESUMO: A escola desempenha papel fundamental na formação de hábitos alimentares e é nesse ambiente que crianças e adolescentes permanecem por expressivo período de tempo diário. A alimentação saudável nas escolas contribui para a aprendizagem, melhorando também as cond ições nutric ionais dos alunos. Com o objetivo de conhec er a aceitabilidade e o índice de adesão à alimentação escolar oferecida aos estudantes do ensino médio deste Instituto foi realizado um estudo de natureza quali-quantitativa. In ic ialmente foram sorteados aleatoriamente $30 \%$ do público alvo, em seguida foi aplic ado um questionário com perguntas objetivas e subjetivas. Calculou-se o valor médio do índice de adesão, analis ando-o através da classific ação relatada no Manual para Aplicação dos Testes de Aceitabilidade no Programa Nacional de Alimentação Escolar, elaborado pelo Centro Colaborador em Alimentação e Nutrição Escolar UNIFESP/2010. Este estudo verific ou alta adesão e boa aceitação à alimentação escolar, no entanto, é necessária a cons trução de um refeitório, bem como a realização de ações de educação alimentar no ambiente escolar, a fim de promover a segurança alimentar e nutric ional dos alunos benefic iados.

Palavras - chave: aceitação, ensino médio, índice de ad esão, refeição escolar

\section{Acceptability and adhesion of the school feeding of IF Sertão of Pernambuco by students in Salgueiro campus}

\begin{abstract}
School plays a fundamental role forming good eating habits since children and teenagers stay for a long amount of time every day in this environment. Healthy eating in the schools contributes for the learning improving the nutritional conditions of students. Aiming to know the acceptability and the adhes ion level of the school feeding offered to the high school students of this Institute, a qualitative and quantitative study was made. Initially 30\% of the target audience was randomly chosen. Then a questionnaire with objective and subjective questions was applied. We calculated the medium average of the adhesion level, analyzing it through the classification reported in the Manual for the Applic ation of Acceptability Test in the School Feeding National Programme, elaborated by the collaborative Center in Feeding and School Nutrition - UNIFESP/2010. This study verified a high adhesion and good acceptance to the school feeding, however, it is necessary to build a school cafeteria, as well as promoting healthy eating education actions in the school environment in order to promote the feeding and nutritional sec urity of the beneficiated students.
\end{abstract}

Key words: acc ep tance, high school, adhesion level, school feeding 


\section{Introdução}

$\mathrm{D}$ e acordo com a Lei $\mathrm{n}^{\circ} 11.346$, de 15 de setembro de 2006 , a a limentação adequada é direito fundamental do ser humano, devendo o poder público adotar as políticas e ações que se façam necessárias para promover e garantir a segurança alimentar e nutricional da população.

Desta forma a alimentação escolar visa garantir a segurança alimentar e nutricional dos alunos da educação básica pública, com acesso de forma igualitária, respeitando as diferenças biológicas entre idades e condições de saúde dos alunos que necessitem de atenção específica e aqueles que se encontram em vulnerabilidade social (LEI N 11.947, 2009).

A alimentação desempenha um papel primordial durante todo o ciclo de vida dos indivíduos. Entre as distintas fases de vida pode-se destacar, por exemplo, a adolescência que se caracteriza por um período em que o jovem apresenta um metabolismo muito mais intenso quando comparado ao adulto (VITOLO, 2008).

O Programa Nacional de Alimentação Escolar (PNAE) visa suprir no mínimo 20\% das necessidades nutricionais durante a permanência na escola, contribuindo para o rendimento escolar, a formação de bons hábitos alimentares e para o aumento na capacidade de aprendizagem dos alunos (BRASIL, 2009).

De acordo com a Resolução/CD/FNDE $\mathrm{N}^{\circ} 38$, de 16 de Julho de 2009, o principal fator para determinar a qualidade dos serviços prestados pelas unidades de alimentação das instituições de ensino é a aceitação da alimentação oferecida aos alunos, a qual é influenciada por vários fatores, entre eles hábitos alimentares da cultura; preferências e aversões alimentares; cond ições fisiológicas do jovem, incluindo alergias, intolerâncias alimentares; além de atitudes, crenças e tabus relativos aos alimentos (VIEIRA \& MARTINS, 2008).

A opção por uma alimentação saudável com destaque para o consumo de frutas e verduras é muito importante, pois ao estimular a adoção de hábitos alimentares saudáveis estará contribuindo para a diminuição de doenças relacionadas à má alimentação, co mo o excesso de peso e as doenças crônicas não transmissíveis, como diabetes tipo 2 e doenças cardiovasculares (MALTA, 2010).

Em 2003 as doenças crônicas não transmissív eis (DCNT) foram responsáveis por $60 \%$ das mortes e incapacidade no mundo, podendo chegar a $73 \%$ das mortes em 2020 . Em 2001, no Brasil, as DCNT foram responsáveis por $62 \%$ das mortes e $39 \%$ das hospitalizações registradas no Sistema Único de Saúde (ACHUTTI \& AZAMBUJA, 2004).

Diante da importância de haver uma alta adesão dos alunos à alimentação escolar, como também uma boa aceitação das preparações servidas estão apresentados neste artigo os resultados desse estudo. $\mathrm{O}$ objetivo foi conhecer a aceitabilidade e o índice de adesão dos beneficiários da alimentação escolar da instituição, possibilitando desta forma verificar o quantitativo de alunos que aderem às refeições oferecidas e verificar a aceitação da alimentação escolar a qual deve respeitar os hábitos alimentares da região, priorizando sempre cardápios saudáveis para o desenvolvimento dos jovens.

\section{Metodologia}

Foi realizado um estudo transversal de natureza quali-quantitativa com os alunos do ensino médio in tegrado aos cursos técnicos do Instituto Federal do Sertão Pernambucano/campus Salgueiro em junho de 2012.

A seleção dos participantes foi feita por amostragem, onde foram sorteados aleatoriamente $30 \%$ do total de estudantes beneficiados com a alimentação escolar fornecida gratuitamente pelo Instituto.

Para a análise do índice de adesão foi utilizado um formulário próprio o qual foi preenchido pela equipe durante uma semana. Nele foram registrados o número de alunos presentes na aula que antecedia o intervalo e o quantitativo de alunos que aderiram (receb eram) à alimen tação escolar. 
(CARLINI; COSTA; MESQUISTA, 2015)

Esta análise foi realizada através da classificação relatada no Manual para Aplicação dos Testes de Aceitabilidade no Programa Nacional de Alimentação Escolar PNAE, elaborado pelo Centro Colaborador em Alimentação e Nutrição Escolar CECANE UNIFESP/2010, no qual possui o cálculo do índice de adesão $\left(\mathrm{IA}=\mathrm{N}^{\circ}\right.$ de alunos que consumiram a refeição $/ \mathrm{N}^{\circ}$ de estudantes presentes na escola x 100), sendo classificado em quatro categorias: alto (acima de 70\%), médio (50 a $70 \%$ ), baixo (30 a $50 \%$ ) e muito baixo (menor que $30 \%$ ).

Para a análise das questões relativas aos motivos da adesão ou não e à aceitação da alimentação por parte dos alunos, foi aplicado um questionário com perguntas objetivas e subjetivas, conforme anexo 1, com 56 estudantes.

O questionário utilizado consta no manual acima referido, o qual foi adaptado para esta pesquisa. Este instrumento foi aplicado a fim de conhecer a adesão, a qualidade e a quantidade da alimentação oferecida, e ainda os alimentos de maior e menor aceitação, a procedência dos alimentos consumidos pelos estudantes (casa, cantina, Instituto) e seus hábitos alimentares.

Inicialmente foi realizado na sala de aula um sorteio para obtenção dos participantes, em seguida foram distribuídos os questionários para os alunos que foram orientados a responder verdadeiramente os quesitos. Os alunos foram auxiliados apenas na leitura do questionário.

Após o preenchimento, os questionários foram recolhidos para posterior análise das informações.

Os dados foram analisados e calculou-se o índice de adesão médio e a aceitabilidade. Para a análise das questões objetivas foi considerada a frequência de respostas dos alunos e nas questõ es subjetivas os resultados foram computados tomando por base a informação central contida nas respostas dos participantes.

\section{Resulta dos e Discussão}

Ao analisar o aspecto quantitativo da adesão dos alunos à alimentação escolar por meio do índice de adesão médio (IAM), este estudo revelou um IAM de 74,43\%, classificado como alto (acima de 70\%), o que caracteriza uma elevada adesão por parte dos beneficiários pelo Programa Nacional de Alimentação Escolar.

Em um estudo realizado por Bleil et al. (2009) foi verificada a adesão à alimentação escolar em 77,3\% dos alunos de instituições públicas de ensino no município de Toledo-PR, semelhante aos resultados encontrados nesta pesquisa.

Outro estudo que também demonstrou elevada adesão foi o de Pegolo e Silva (2010) que ao analisar o índice de adesão de escolares matriculados na rede pública de ensino de Piedade-SP verificaram um resultado igual a $81,3 \%$.

$\mathrm{Na}$ tabela 1, verifica-se que apenas $1,79 \%$ dos alunos não costuma comer a alimentação oferecida, justificando lanchar na cantina da instituição. A maioria $(67,27 \%)$ registrou que costuma comer a alimentação escolar 5 dias na semana.

Em relação à aceitação, $61,82 \%(n=34)$ afirmaram gostar de todas as preparações oferecidas na alimentação e 38,18\% (n=21) alegaram não gostar de algumas preparações, sendo as mais mencionadas, vitamina de mamão $(n=5)$, farofa de bolacha $(n=3)$, frutas $(n=2)$, verduras $(n=2)$, frango $(n=2)$, macarrão $(\mathrm{n}=2)$.

Entretanto, todos os participantes que já experimentaram a alimentação escolar relataram que as preparações que mais gostam são cachorro quente, baião de dois com carne, arroz com frango, macarronada, saladas, frutas, vitaminas de frutas, fígado e sucos.

A maioria dos alunos $(85,71 \%)$ ainda informou através do preenchimento do questionário, não possuírem nenhuma alergia e/ou intolerância alimentar. 
(CARLINI; COSTA; MESQUISTA, 2015)

Tabela 1. Distribuição das respostas dos alunos quanto à adesão e à aceitação da alimentação oferecida pelo IF Sertão PE/campus Salgueiro.

\begin{tabular}{|c|c|c|}
\hline Perguntas e respostas & $n$ & $\%$ \\
\hline \multicolumn{3}{|l|}{$\begin{array}{l}\text { Você costuma comer a alimentação ofe recida na merenda do } \\
\text { instituto? }\end{array}$} \\
\hline Sim & 55 & 98,21 \\
\hline Não & 1 & 1,79 \\
\hline Total & 56 & 100 \\
\hline Perguntas e respostas & $n$ & $\%$ \\
\hline \multicolumn{3}{|l|}{$\begin{array}{l}\text { Quantos dias por semana você costuma comer a alimentação } \\
\text { oferecida pelo Instituto? }\end{array}$} \\
\hline 1 dia na semana & 0 & 0 \\
\hline 2 dias na semana & 2 & 3,64 \\
\hline 3 dias na semana & 4 & 7,27 \\
\hline 4 dias na semana & 12 & 21,82 \\
\hline 5 dias na semana & 37 & 67,27 \\
\hline Total & 55 & 100 \\
\hline Perguntas e respostas & $n$ & $\%$ \\
\hline \multicolumn{3}{|l|}{$\begin{array}{l}\text { Você gosta da alimentação oferecida no Instit uto no ho rário do } \\
\text { recreio? }\end{array}$} \\
\hline Sim, gosto de todas as preparações oferecidas na alimentação & 34 & 61,82 \\
\hline Não gosto de algumas & 21 & 38,18 \\
\hline Não gosto de nenhuma & 0 & 0 \\
\hline Total & 55 & 100 \\
\hline
\end{tabular}

Quanto à qualidade, quantidade e local onde é servida a refeição observa-se na tabela 2 que $62,50 \%$ dos participantes marcaram que a temperatura das preparações está sempre boa, porém $35,71 \%$ relataram que às vezes algumas preparações, principalmente o macarrão, estão frias ou muito quentes. Este resultado provavelmente foi devido à inexistência provisoriamente de um balcão térmico para manter aquecidas as preparações, sendo a equipe responsável pela produção da alimentação escolar orientada a produzir os alimentos próximos ao horário do lanche. Algumas preparações como a macarronada sofrem rápidas variações de temperatura se não estiverem sendo oferecidas no equipamento adequado.
A quantidade da alimentação servida foi classificada como boa por $60,71 \%$ do público, sendo caracterizada como insuficiente por $39,29 \%$ dos alunos.

Quando foram questionados sobre o local onde é servido o lanche, a maioria respondeu que o local não é confortável e $62,07 \%$ justificaram que é porque não tem lugar para todos sentarem. Ainda sobre esta questão, 6,89\% dos alunos justificaram o desconforto por outros motivos, 1 registrou ser muito longe e o outro que poderia ser "de uma forma diferente". Com o intuito de sanar este problema, há previsão de que um refeitório será construído para que os alunos possam realizar suas refeições em um ambiente adequado. 
(CARLINI; COSTA; MESQUISTA, 2015)

Tabela 2. Distribuição das respostas dos alunos quan to à temperatura, à quantidade da alimentação oferecida pelo IF Sertão PE/campus Salgueiro e o local onde é servida.

\begin{tabular}{|c|c|c|}
\hline Perguntas e respostas & $n$ & $\%$ \\
\hline \multicolumn{3}{|c|}{$\begin{array}{l}\text { Na sua opinião a temperatura da alimentação servida no } \\
\text { recreio é: }\end{array}$} \\
\hline Sempre boa & 35 & 62,50 \\
\hline Às vezes é boa & 20 & 35,71 \\
\hline Nunca é boa & 1 & 1,79 \\
\hline Total & 56 & 100 \\
\hline Perguntas e respostas & $n$ & $\%$ \\
\hline \multicolumn{3}{|c|}{$\begin{array}{l}\text { Na sua opinião a quantidade de comida que é servida na } \\
\text { alimenta ção é: }\end{array}$} \\
\hline Muita [exagerada] & 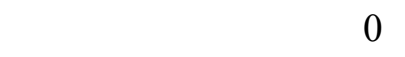 & 0 \\
\hline Boa [suficiente] & 34 & 60,71 \\
\hline Pouca [insuficiente] & 22 & 39,29 \\
\hline Total & 56 & 100 \\
\hline Perguntas e respostas & $n$ & $\%$ \\
\hline \multicolumn{3}{|c|}{$\begin{array}{l}\text { Na sua opinião o local onde é servida a alimenta ção é } \\
\text { confortável? }\end{array}$} \\
\hline Sim & 27 & 48,21 \\
\hline Não & 29 & 51,79 \\
\hline Total & 56 & 100 \\
\hline Perguntas e respostas & $n$ & $\%$ \\
\hline \multicolumn{3}{|c|}{ Se você acha que o local não é confortável, diga-me o motivo. } \\
\hline Não tem mesa & 8 & 27,59 \\
\hline É sujo & 0 & 0 \\
\hline E barulhen to & 1 & 3,45 \\
\hline Outro(s) & 2 & 6,89 \\
\hline Total & 29 & 100 \\
\hline \multicolumn{3}{|c|}{$\begin{aligned} \text { De acordo com os resultados expostos } & \text { têm cheiro estranho }(\mathrm{n}=4) \text {. Como nenhum } \\
\text { na tabela } 3 \text { a maioria dos alunos relatou gostar } & \text { estudante marcou a opção que o copo/caneca é } \\
\text { dos talheres, copos e pratos utilizados para } & \text { sujo, o odor deve ter origem no material de } \\
\text { servir a alimentação escolar. } & \text { fabricação. Com relação aos pratos utilizados } \\
\text { Já a minoria respondeu não gostar dos } & \text { para servir a refeição, quatro alunos } \\
\text { talheres oferecidos na alimentação porque não } & \begin{array}{l}\text { informaram que não gostam de comer no prato } \\
\text { gostam de comer comida sólida com colher. Os }\end{array} \\
\text { que marcaram que não gostam do tipo de } & \text { O intervalo escolar para descanso e } \\
\text { copo/caneca responderam que não gostam dos } & \text { alimentação é de } 15 \text { minutos, sendo } \\
\text { utensílios de plástico }(n=3) \text { e que os mesmos } & \text { considerado curto por } 91,07 \% \text { dos estudantes. }\end{aligned}$} \\
\hline
\end{tabular}

Tabela 3. Distribuição das respostas dos alunos quanto aos talheres, aos copos, aos pratos e ao tempo do intervalo para a alimentação oferecida pelo IF Sertão PE/campus Salgueiro. 
(CARLINI; COSTA; MESQUISTA, 2015)

\begin{tabular}{|c|c|c|}
\hline Perguntas e respostas & $n$ & $\%$ \\
\hline \multicolumn{3}{|l|}{ Você gosta dos talheres oferecidos na alimentação? } \\
\hline Sim & 52 & 92,86 \\
\hline Não & 4 & 7,14 \\
\hline Total & 56 & 100 \\
\hline Perguntas e respostas & $n$ & $\%$ \\
\hline \multicolumn{3}{|l|}{ Você gosta do tipo de copo/ca neca usado na alime ntação? } \\
\hline Sim & 49 & $\mathbf{8 7 , 5 0}$ \\
\hline Não & 7 & 12,50 \\
\hline Total & 56 & 100 \\
\hline Perguntas e respostas & $n$ & $\%$ \\
\hline \multicolumn{3}{|l|}{ Você gosta do tipo do prato no qual é servida a alimentação? } \\
\hline Sim & 52 & 92,86 \\
\hline Não & 4 & 7,14 \\
\hline Total & 56 & 100 \\
\hline Perguntas e respostas & $n$ & $\%$ \\
\hline \multicolumn{3}{|l|}{ Na sua opin iã o o tempo disponível para comer a alime nta ção é: } \\
\hline Curto & 51 & 91,07 \\
\hline Suficiente & 5 & 8,93 \\
\hline Longo & 0 & 0 \\
\hline Total & 56 & 100 \\
\hline
\end{tabular}

Outra avaliação importante realizada $\mathrm{R} \$ 10,00$. Os produtos que eles informaram neste estudo foi conhecer os hábitos mais consumirem nestes estabelecimentos são alimentares dos escolares através da salgados, pastéis, tortas, lasanhas, refrigerantes, procedência dos alimentos que são consumidos doces, sucos industrializados, sorvetes, na instituição de ensino. Apesar dos resultados bombons, hambúrgueres, mistos e pipocas.

mostrarem alta adesão à alimentação escolar, a Quando foram questionados sobre a partir da tabela 4 nota-se a visível adoção de motivação para comprar lanche na cantina, hábitos alimentares inadequados, pois $76,79 \% \quad 37,21 \%$ responderam que é devido a não gostar relataram comprar alimentos na cantina.

Os alunos que compram lanche na cantina do IF ou em outros locais informaram que costumam gastar por dia entre $\mathrm{R} \$ 2,00$ e da alimentação oferecida no dia e 30,23\% informaram que compram lanche na cantina da escola mesmo quando comem a alimentação escolar.

Tabela 4. Dis tribuição das respostas dos alunos quanto à compra de lanches na cantina do IF Sertão $\mathrm{PE} /$ campus Salgueiro.

\begin{tabular}{lcc}
\hline Perguntas e respostas & $\boldsymbol{n}$ & $\boldsymbol{\%}$ \\
\hline Você costuma comprar alimentos na cantina do institu to? & 43 & $\mathbf{7 6 , 7 9}$ \\
Sim & 13 & 23,21 \\
Não & $\mathbf{5 6}$ & $\mathbf{1 0 0}$ \\
Total & & \\
\hline & $\boldsymbol{n}$ & $\boldsymbol{\%}$ \\
\hline Perguntas e respostas
\end{tabular}

Quantos dias por se mana você costuma comprar os alimentos 
(CARLINI; COSTA; MESQUISTA, 2015)

\begin{tabular}{lcc}
\hline na cantina do instituto? & 10 & 23,26 \\
1 dia na semana & 18 & $\mathbf{4 1 , 8 6}$ \\
2 dias na semana & 8 & 18,60 \\
3 dias na semana & 3 & 6,98 \\
4 dias na semana & 4 & 9,30 \\
5 dias na semana & $\mathbf{4 3}$ & $\mathbf{1 0 0}$ \\
Total
\end{tabular}

\begin{tabular}{|c|c|c|}
\hline \multicolumn{3}{|c|}{$\begin{array}{l}\text { Pergun tas e respostas } \\
\text { Quando você costuma comprar alimentos na cantina da escola? }\end{array}$} \\
\hline \multicolumn{3}{|c|}{ Quando você costuma comprar alimentos na cantina da escola? } \\
\hline Quando não come a alimentação oferecida no instituto & 12 & 27,91 \\
\hline Quando não traz lanche de casa & 2 & 4,65 \\
\hline Quando não gosta da alimentação oferecida no dia & 16 & 37,21 \\
\hline Mesmo quando come a alimentação, compra na cantina & 13 & 30,23 \\
\hline Total & 43 & 100 \\
\hline $\begin{array}{l}\text { A aquisição de alimentos } \\
\text { comercializados na cantina por parte dos } \\
\text { estudantes é um fato preocupante, pois nem }\end{array}$ & $\begin{array}{l}\text { diversid ade } \\
\text { ambiental, } \\
\text { socialmente } \\
\mathrm{N}^{\circ} 11.947,2\end{array}$ & $\begin{array}{l}\text { cultural e que sejam } \\
\text { cultural, econômica e } \\
\text { sustentáveis." (LEI } \\
\text { 009) }\end{array}$ \\
\hline
\end{tabular}
Hábitos alimentares inadequados aliados à diminuição progressiva da atividade física convergem para o aumento no número de casos de obesidade em todo o mundo, sendo um fator de risco para o desenvolvimento de doenças crônicas não transmissíveis como hipertensão arterial, diabetes mellitus e doenças cardiovasculares (MARIATH, 2007).

A partir dos resultados observados percebe-se uma boa aceitação da alimentação escolar gratuita oferecida na instituição. Verifica-se, porém, a necessidade de ações de educação nutricional que orientem os estudantes sobre a escolha de alimentos saudáveis explicando os motivos pelos quais devem adotar hábitos alimentares adequados.

Uma das diretrizes da alimentação escolar é a inclusão da educação alimentar e nutricional no processo de ensino e aprendizagem, abordando o tema alimentação e nutrição e o desenvolvimento de práticas saudáveis de vida, na perspectiva da segurança alimentar e nutricional (LEI N¹1.947, 2009).

\footnotetext{
"A segurança alimentar e nutric ional consiste na realização do direito de todos ao acesso regular e permanente a alimentos de qualidade, em quantidade sufic iente, sem comprometer o acesso a outras necessidades essenciais, tendo como base práticas alimentares promotoras de saúde que respeitem a
}

\section{Conclusões}

Com base nos resultados obtidos, foi observada uma alta adesão pelos beneficiários da alimen tação escolar.

$\mathrm{Na}$ opinião dos alunos, alguns aspectos relacionados à alimentação podem ser melhorados. Entre eles, destaca-se a temperatura de algumas preparações, que não está sempre boa; e o local onde é servida a alimentação, que foi considerado desconfortável. Estes fatos relacionam-se à inexistência de um refeitório, sugerindo-se aos gestores a urgente implantação do referido espaço.

Este estudo revelou ainda que os alunos que adquirem produtos na cantina, geralmente optam por alimentos não saudáveis, sendo constatada a necessidade de ações de educação nutricional para que a partir dos conhecimentos adquiridos os estudantes possam fazer suas escolh as de forma consciente.

Verifica-se, portanto, a importância de acompanhar e investigar constantemente a aceitação e adesão à alimentação gratuita oferecida aos alunos, uma vez que, através dos controles realizados é possível diminuir desperdícios, conhecer os reais motivos da adesão ou não à alimentação escolar, a fim de 
(CARLINI; COSTA; MESQUISTA, 2015)

corrigir distorções, e promover a segurança alimentar e nutricional dos alunos beneficiados.

\section{Agradecimentos}

À instituição, por proporcionar esta oportunidade de estudo;

Aos alunos, por participarem da pesquisa;

À professora de inglês da instituição, Roberta Guimarães de Godoy e Vasconcelos, por nos auxiliar na produção do resumo em inglês.

\section{Referências}

ACHUTTI, A. \& AZAMBUJA, M.I.R. Doen ças crônicas não transmissíveis no Brasil: repercussões do modelo de atenção à saúde sobre a seguridade social. Ciência \& Saúde Coletiva, 9(4):833-840, 2004.

BLEIL, R.A.T.; SALAY, E. \& SILVA, M.V. Adesão ao Programa de Alimentação Escolar por Alunos de Instituições Públicas de Ensino no Município de Toledo, PR. Segurança Alimentar e Nutricional, Campinas, 16(1): $65-$ 82, 2009.

BRASIL. Lei $\mathrm{n}^{\circ} 11.346$, de 15 de setembro de 2006. Cria o Sistema Nacional de Segurança Alimentar e Nutricional - SISAN com vistas em assegurar o direito humano à alimentação adequada e dá outras providências.

BRASIL. Lei $\mathrm{n}^{\circ} 11.947$, de 16 de junho de 2009. Dispõe sobre o atendimento da alimentação escolar e do Programa Dinheiro Direto na Escola aos alunos da educação básica e dá outras providências.

BRASIL. Resolução/CD/FNDE n ${ }^{\circ}$ 38, de 16 de julho de 2009. Dispõe sobre o atendimento da alimentação escolar aos alunos da educação básica no Programa Nacional de Alimentação Escolar - PNAE, Brasília DF. [Acesso em Jul 2012]. Disponível em: www.fnde.gov.br

FUNDO NACIONAL DE DESENVOLVIMENTO DA EDUCAÇÃO. Manual para aplicação dos testes de aceitabilidade no PNAE. Disponível em: $<$ http://www.fnde.gov.br/index.php/aealimentacao-e-nutricao $>$. Acesso em 29 Ago. 2011.

MALTA, Deborah Carvalho et al . Prevalência de fatores de risco e proteção de doenças crônicas não transmissíveis em adolescentes: resultados da Pesquisa Nacional de Saúde do Escolar (PeNSE), Brasil, 2009. Ciênc. saúde coletiva, Rio de Janeiro, v. 15, supl. 2, Oct. 2010 .

MARIATH, Aline Brandão et al . Obesidade e fatores de risco para o desenvolvimento de doenças crônicas não transmissíveis entre usuários de unidade de alimentação e nutrição. Cad. Saúde Pública, Rio de Janeiro, v. 23, n. 4, Apr. 2007.

PEGOLO, G.E. \& SILVA, M.V. Consumo de energia e nutrientes e a adesão ao Programa Nacional de Alimentação Escolar (PNAE) por escolares de um município paulista. Segurança Alimentar e Nutricional, Campinas, 17(2): 50$62,2010$.

VIEIRA, P.P. \& MARTINS, R.C.B. Aceitabilidade da alimentação escolar no ensino fundamental da cidade de Adamantina/SP. Boletim das Faculdades Adamantinenses Integradas, Adamantina, n. 2, p. 6-7, 2008.

VITOLO, M.R. Nutrição da gestação ao envelhecimento. Rio de Janeiro, Rubio, 2008. $628 \mathrm{p}$. 